European Union's peripheral countries and its neighbours to the east so as to increase significantly Europe's overall strength and potential to adjust to world competition.

The remarks by J.C. Phillips on the value and effectiveness of small-scale science as opposed to large-scale projects must be endorsed. Europe should build efficient networks of research groups and laboratory facilities, taking full advantage of the richness and diversity existing in the different countries. Recent European Union networking and mobility programmes should be encouraged. In parallel, a programme could be launched to create a network of strategic medium-size research facilities in the various European regions, with adequate interfaces with industry and conditions that promote an efficient transfer of scientific knowledge to new applications.

I also support the need to introduce new approaches in teaching and training science at the university level. In particular, we must learn how to integrate high-level science with technologically oriented knowledge in order to formulate "science with a purpose". An overly academic and removed science, although very formative and of universal validity, is generally ineffective if it is not complemented by (simultaneous) information on the applied aspects of science and the basic principles behind modern technology. Students should also be trained in the use of science for solving concrete problems, in an open environment and through interaction with non-academic people. As J.C. Phillips pointed out, most of industrial practice consists not of profound inventions and carefully prepared discoveries, but of quick and

\title{
We Need the Whole of Physics
}

\section{J.T. Devreese from the University of Antwerp points out the danger of trivializing parts of physics.}

\section{J.T. Devreese}

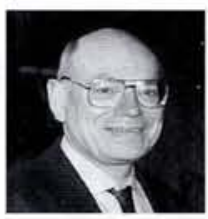

The analysis by J.C. Phillips at CMD-EPS ' 96 of solid-state physics was certainly stimulating. But remarks such as "high-energy physics has been dead for 30 years" and a strong opposition to the SSC that were perhaps intended to enliven the discussion. They should be taken cum maximo grano salis. First of all, high-energy physics remains a vigorous field with some of the best minds at work. In the period referred to we have seen the discovery of the J/psi, of the $\mathrm{W}$ and $\mathrm{Z}$ bosons and of the top quark, as well as the development of the standard model of particle physics which constitutes a great step forward towards realizing the dream of unification. These achievements have not only contributed to our understanding of the "building blocks" of Nature but also shed light on the evolution of the early universe. Think of the marvellous "first three minutes" of Weinberg; the fact that the structure and dynamics of the universe is correlated with the fine details of elementary particle physics is a fundamental insight with a major cultural dimension. Furthermore, the methods of theoretical high-energy physics have been most fruitful for condensed-matter physics, notably

timely incremental steps which either enable new methods, or which control quality to stabilize and improve the yield of older makeshift path integrals and Feynman diagrams, and concepts such as scaling, universality and symmetry breaking have been useful in many fields of physics. The unique tool of synchrotron radiation, so dear to solid-state physicists, is a by-product of high-energy physics.

We need all of physics: the beauty of celestial mechanics, the depths of phase transitions, the excitement of understanding the hydrostatic paradox or the colour of the sky, the joy of calculating the Lamb shift or measuring the $21 \mathrm{~cm}$ line. It is counterproductive to minimize the significance of areas other than one's own. It is also hard to see how to attract students by trivializing the value of parts of physics. What we must convey is the richness of the entire subject and, indeed, of the whole of science.

So-called small science is indeed invaluable: the work of Müller and Bednroz on high$\mathrm{T}_{c}$ superconductivity was realized with modest budgets, and the same is true for the BinnigRohrer scanning tunnellng microscope. On the other hand, detection of the quantum Hall effect was facilitated by the availability of high magnetic fields. So we should open up the full potential of both large and small science.

methods. Here, acute awareness and mixed experience (science/technology) are crucial factors for success.

\section{W IMPERIAL COLLEGE of Science, Technology \& Medicine Professional Development Courses \\ Neural Networks: \\ Basics, Applications \& Futures \\ 18-20 September 1996}

A modular 3-day programme for all with interests in this important field. In the first two days, the basics and applications, both industrial and commercial, of neural networks will be discussed. The final day will be a seminar on the 'Future of Neural Networks', with international authorities including Igor Aleksander, Shun-Ichi Amari, John Hopfield, Teuvo Kohonen and John Taylor.

\section{Safety Critical Systems \\ 16- 20 September 1996}

Designed for all those who require an understanding of the approach and methods used to generate software in safety critical systems, whatever the application. It will be of interest both to software engineers, who need to familiarise themselves with techniques, and to those who specify or manage projects in which safety issues are a concern. Participants will be taught how to use software tools by working, in groups, through small projects in SCS development.

\section{Microsystems Technology}

\section{$23-25$ October 1996}

An inter-disciplinary programme covering the science, technology and application of microsystems - i.e. sensors, actuators and other 3D-structures of emerging importance. Topics covered will include materials, fabrication, devices and applications.

Further details from: Sally Verkaik, Continuing Education Centre, Imperial College, Level 5 Sherfield Building, Exhibition Road, London SW7 2AZ, UK.

Tel: +44 (0)171 594 6882/1; Fax: +44 (0)171 5946883 E-mail: cpd@ic.ac.uk

\section{BERGISCHE UNIVERSITÄT - GESAMTHOCHSCHULE WUPPERTAL}

\section{The institute for Materials Science}

Invites applications for newly created, tenured faculty position:

\section{FULL PROFESSOR (Universitätsprofessor C4) of Theoretical Physics}

The successful applicant will be appointed to the Physics Faculty and is expected to build up and to lead a group devoted to the theoretical analysis of new materials or novel condensed matter phenomena. The applicants are expected to meet the corresponding standards of scientific and academic qualification and experience. Besides carrying out a successful research program in cooperation with existing groups in the Institute for Materials Science this professor is expected to participate in teaching the curricula in physics and materials science.

Applicants must satisfy the legal requirements of the state of Northrhine-Westfalia for the above appointment, i.e., Habilitation or equivalent scientific merits or degrees. They should send their curriculum virae, a summary of their scientific career including teaching experience, and a list of publications by November 10 , 1996 to the Director of the Institute for Materials Science,

Prof. Dr. S. Dietrich, Fachbereich Physik,

Bergische Universität - Gesamthochschule Wuppertal,

D-42097 Wuppertal, Germany 